\title{
Patientenpräferenz von Inhalern
}

\author{
Inhalation Therapy, Patient's Perspective
}

Autor

Institut

\section{A. Gillissen}

Klinik für Lungen- und Bronchialmedizin, Klinikum Kassel eingereicht $\quad 23.5 .2014$

akzeptiert 10.6.2014

\section{Bibliografie}

DOI http://dx.doi.org/

10.1055/s-0034-1377573

Online-Publikation: 17.9.2014

Pneumologie 2014; 68: 727-736

(c) Georg Thieme Verlag KG

Stuttgart · New York

ISSN 0934-8387

Korrespondenzadresse
Prof. Dr. Adrian Gillissen
Klinik für Lungen- und
Bronchialmedizin
Klinikum Kassel,
Kassel School of Medicine
Möncheberg-Str. 41-43
34125 Kassel
adrian.gillissen@klinikum-kassel.
de
www.klinikum-kassel.de

www.klinikum-kassel.de

\section{Zusammenfassung \\ $\nabla$}

Standbein der Medikamentenapplikation bei Asthma und COPD ist die Inhalation. Der Vorteil ist die Therapie direkt am Ort des Geschehens. Allerdings steigt durch die Fülle an immer wieder neuen auf dem Markt angebotenen Inhalern eher die Verwirrung, als dass diese als Möglichkeit einer differenzierten Applikationsform wahrgenommen werden. Die korrekte, Patienten-bezogene Verschreibung des richtigen Inhalers wird für den verschreibenden Arzt und den Anwender zu einer schwierigen und nur schwerlösbaren Aufgabe. Der Aspekt der korrekten für den Patienten passenden Inhalerauswahl wird in Asthmaund COPD-Leitlinien kaum oder gar nicht berücksichtigt, obwohl bekannt ist, dass eine komplizierte Inhalertechnik, eine fehlende oder unzureichende Einweisung, Fehler bei der Anwendung und die fehlende Berücksichtigung der Patientenbedürfnisse zu einer ineffektiven Medikamentenapplikation, einer schlechten Patientenadhärenz und damit zu einer unzureichenden Kontrolle der zu therapierenden obstruktiven Atemwegserkrankung führen. Es wird hier eine Übersicht über die wichtigsten Inhalertypen gegeben und die Beziehung zwischen der Patientenakzeptanz auf der einen und der Fehlerhäufigkeit auf der anderen Seite erläutert. Grundsätzlich gilt, je besser der Patient seinen Inhaler akzeptiert und in der Folge auch mit seiner Technik zurechtkommt, desto besser ist der Therapieerfolg.

\section{Einleitung}

\section{$\nabla$}

Die Standardapplikationsform von Medikamenten bei Asthma und der COPD (chronisch-obstruktive Lungenerkrankung) ist die inhalative Gabe. Dafür stehen u.a. druckluft- oder ultraschallbetriebene Inhalatoren, Dosieraerosole und Trockenpulverinhalatoren zur Verfügung. Der offensichtliche Vorteil einer inhalativen Medika-

\section{Abstract \\ $\nabla$}

The cornerstone of drug application in obstructive pulmonary diseases is by inhalation as advocated by all treatment guidelines. Today, there exists an extensive choice of inhalers and new models. Numerous inhaler types are constantly being developed and introduced into the market making the "correct" selection a wearying task for the physician as well as for the patient. Improvement in the management of this aspect, which is often neglected in its importance in daily practice as well as in guidelines, is therefore reliant not only on the medications themselves but also on their effective delivery. Complicated inhaler mechanics and poor inhaler technique which do not meet the patient requirements and desires contribute to poor compliance eventually leading to poor disease control. In this review, the importance of inhaler acceptance and the degree of satisfaction with different inhaler types are discussed. This paper focuses on the behavioral and attitudinal evidence that the acceptance of the inhaler is linked to patient compliance, and its linkage on health and patient-reported outcomes. 
riger und belastender als eine Tabletteneinnahme. Die korrekte Anwendung muss dem Patienten erst erklärt werden. Es sind sowohl seitens der korrekten Atemtechnik als auch bzgl. der technischen Anwendung des Inhalers viele Anwendungsfehler möglich. Die Therapietreue ist im Vergleich zur Tabletteneinnahme schlechter, und die große Auswahl von Inhalationssystemen führt nicht selten zu einer großen Unsicherheit bei den Ärzten, welcher Inhaler sich für die Therapie am besten eignet, und bei den Patienten zu Unsicherheiten in der Anwendung [2,3]. Gerade die große Auswahl und die als kompliziert empfundenen physikalischen Grundlagen der Aerosolmedizin sowie die technischen Inhalerspezifika fördern eher die Verwirrung und die Unsicherheit, als dass sie die zielgerichtete und Patienten-adaptierte Verordnung und Anwendung fördern. Ziel einer jeden Inhalationstherapie sollte daher ein Inhaler sein, mit dem der Patient zurechtkommt, den er wie verordnet regelhaft anwendet und den er „mag“.

In dieser Übersicht soll ein Einblick darüber gegeben werden, welche Präferenzen unsere Patienten in Bezug auf die ihnen verordneten Inhalationsgeräte haben, ein Aspekt, der in der Praxis aus vielerlei Gründen oft unberücksichtigt bleibt.

\section{Einfluss der Inhalerauswahl}

\section{$\nabla$}

Asthma- und COPD-Therapieleitlinien, wie z. B. GINA (global initiative for asthma) und GOLD (global initiative for COPD) empfehlen zwar in Bezug auf die Inhalerauswahl, dass der Inhaler portabel, einfach anzuwenden, ohne eine externe Energiequelle zu betreiben, eine geringe Kooperation seitens des Patienten erfordern, umweltverträglich, konstante Dosisabgabe mit geringen Dosisschwankungen und ein Zählwerk haben soll, aber klammern die Berücksichtigung individueller Patientenspezifika aus, denen wir tagtäglich im medizinischen Alltag begegnen $[4,5]$. Die Leitlinie der British Thoracic Society empfiehlt zusätzlich die Patienten zu befragen und individuelle Präferenzen bzgl. der Inhalerauswahl und deren Fähigkeit im korrekten Umgang mit dem Inhaler zu berücksichtigen [6]. Eine ähnliche Empfehlung wurde auch von der American College of Chest Physicians und der American Society of Asthma, Allergy, and Immunology publiziert [7]. In der betont technisch verfassten Empfehlung für die Auswahl von Inhalationssystemen zur Medikamentenverabreichung der Arbeitsgruppe Aerosolmedizin der DGP (Deutsche Gesellschaft für Pneumologie und Beatmungsmedizin) wird empfohlen, dass „falls mehrere Inhalationssysteme theoretisch anwendbar erscheinen, sollten Patientenpräferenzen berücksichtigt werden“. Diese Empfehlung bezieht sich also nicht auf die Situation, wenn nur ein einziger Inhaler verschrieben wird [1]. Vor Verschreibung einer Inhalationstherapie sind folgende Aspekte zu berücksichtigen:

- Ist der Patient fähig, ein suffizientes Inhalationsmanöver auszuführen, und ist der inspirative Flow ausreichend? Wenn ja, empfiehlt sich ein Trockenpulverinhalationssystem (DPI = dry powder inhaler). Wenn nein, wäre die Verschreibung eines Treibgas-betriebenen Inhalers (pMDI = pressured metered dose inhaler) evtl. zusammen mit einem Spacer oder eine Verneblertherapie sinnvoll.

- Bestehen Koordinationsprobleme zwischen Auslösung eines pMDI und der gleichzeitigen tiefen Inspiration, wäre entweder ein DPI, ein Vernebler, ein MDI zusammen mit einem Spacer oder ein Inhaler ratsam, der erst bei einem ausreichenden inspiratorischen Atemflow die Medikamentenmenge freigibt (Atemzugtriggerung).

- Müssen täglich mehrere inhalative Medikamente eingenommen werden, ist es zweckmäßig, die Medikamentenmenge durch die Verordnung eines Kombinationspräparates zu minimieren. Zudem sollten zwecks einfacher Handhabung nicht verschiedene Inhalertypen verschrieben werden. Andernfalls ist mit einer zunehmenden Fehlerhäufigkeit, einer Verschlechterung der Patientenmitarbeit (Adhärenz) und einem Qualitätsverlust in der inhalativen Therapie zu rechnen. Van der Palen et al. (1999) beobachteten, dass Asthmapatienten mit mehr als einem Inhaler ca. 10\% mehr Fehler bei der Anwendung machten als diejenigen, die nur ein Gerät oder Kombinationsgerät verschrieben bekamen, wobei sie mit einem DPI besser zurecht kamen als mit einem pMDI [8].

- Nur wenn der Patient seine(n) Inhaler akzeptiert, damit überprüfbar zurechtkommt und den Effekt einer erfolgreichen inhalativen Therapie auch verspürt, ist anzunehmen, dass er ihn regelmäßig und korrekt anwendet [2,9-12].

- Ungeachtet des verschriebenen Inhalertyps ist immer eine Inhalerschulung notwendig, und der Patient muss auch umgekehrt zeigen, dass er den Inhaler richtig anwenden kann, denn Fehler sind leider die Regel und nicht die Ausnahme [13-15].

- Eine höhere Patientenzufriedenheit mit dem Inhaler ist mit einer höheren Lebensqualität, einer geringeren Exazerbationsrate, weniger Arztbesuchen und einer besseren Schlafqualität verbunden, wie Small et al. 2011 bei Asthmapatienten nachweisen konnten. Als Grund wird eine bessere Patientenadhärenz und in der Folge eine bessere Asthmakontrolle angenommen [16].

Ärzte lassen sich in Bezug auf die Verschreibung des Inhalers primär leiten durch: a) die für die Therapie notwendige Substanzgruppe, b) den dafür zur Verfügung stehenden Inhalertyp und c) ihren medizinischen, ggf. auch technischen Kenntnisstand und d) ihre persönlichen Präferenz [17]. Bei der Fülle der heute angebotenen Inhalern wird es wahrscheinlich noch nicht einmal dem Facharzt möglich sein, diese Vielfalt, deren technische Details und deren Vor- und Nachteile für sich selbst abschätzen und schlussendlich ihren Patienten vorzustellen zu können. Die Auswahl wird also zwangsläufig nach individuellen Arztvorlieben und rein praktischen Vorgehensweisen vorgenommen werden. Der Patient wird sich der vom Arzt präferierten Inhalerauswahl unterwerfen müssen, zumal er die Alternativen nicht kennt und aus Zeit- bzw. Praktikabilitätsgründen höchstwahrscheinlich auch nicht kennenlernen wird und kann.

\section{Was wünscht der Patient? \\ $\nabla$}

Gefragt, welche Faktoren die eigene Therapietreue am meisten beeinflussen bzw. welche Faktoren in Bezug auf die Therapie am wichtigsten seien, beantworteten amerikanische Asthmapatienten entsprechend der folgenden Reihenfolge [18]:

1. bessere Symptomenkontrolle durch die inhalative Therapie

2. langwirkende Symptomenkontrolle

3. Reduktion der Notfallmedikation

4. Reduktion zusätzlich notwendiger Medikamente, um das Asthma zu kontrollieren

5. Verbesserung des Allgemeinbefindens und damit verbundene Steigerung der Lebensqualität

6. schneller Wirkungsbeginn 


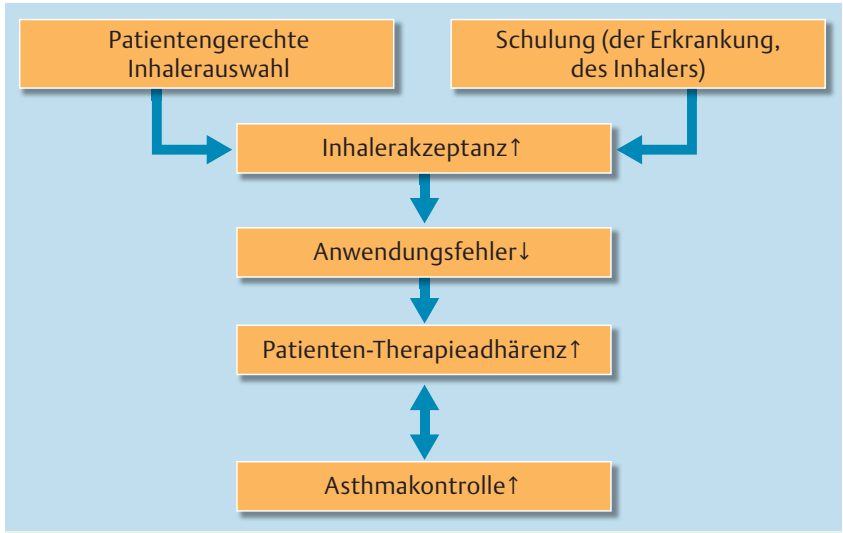

Abb. 1 Idealisierte Abhängigkeiten von Inhalerauswahl, -akzeptanz seitens des Patienten, Fehler bei der inhalativen Anwendung und Therapieadhärenz.

In der nach eigenen Angaben schlecht adhärenten Asthma-Subgruppe wurden etwas andere Gründe bzw. eine andere Gewichtung für die Nicht-Adhärenz angegeben:

1. Verbesserung der Lebensqualität

2. schneller Wirkungsbeginn

3. lange Symptomenkontrolle

4. effektivere Symptomenkontrolle

5. Reduktion der Notfallmedikation

6. Sicherheitsaspekte der langfristigen Medikamenteneinnahme In erster Linie ist für den Patienten der spürbare Medikamenteneffekt wichtig [19]. Da der Medikamenteneffekt aber nur zu erwarten ist, wenn die inhalative Anwendung, wie vom Arzt verordnet, korrekt angewendet und die Therapie auch vom Patienten akzeptiert wird, kommt der Inhalerakzeptanz eine hohe Bedeutung zu. Umgekehrt gibt es eine lineare Korrelation zwischen einer schlechten Inhalertechnik und einer schlechten Patientenadhärenz. Eine gute Inhalerakzeptanz ist wiederum ein Indikator für die Fähigkeit, dass die inhalative Therapie korrekt angewendet wird, was wiederum mit einer guten Patienten-Therapieadhärenz korreliert $[16,20,21]$ ( Abb.1). Im positiven Fall sinken die Exazerbationen, die Häufigkeit der notfallmäßigen Krankenhauseinweisungen, Arztvorstellungen, und die Patienten geben an, dass sie nachts weniger Asthmaanfälle erleiden würden [16]. Patienten haben eine klare Vorstellung davon, welche Charakteristika ein idealer Inhaler haben sollte. Serra-Batlles et al. führten eine Befragung an Asthma- und COPD-Patienten durch und fanden im Wesentlichen, dass die einfache Handhabbarkeit und ein Dosiszähler, durch den die noch verbliebene Medikamentenmenge ablesbar oder errechenbar ist, wichtig sind [22, 23] ( Tab.1).

\section{Inhaler-Präferenz}

Das Ergebnis von Studien zu den Patientenpräferenzen eines bestimmten Inhalers scheint stark durch den Sponsor und das Design der Studie geprägt zu sein. Es liegt in der Natur der Fragestellung, dass solche Device-Studien im Gegensatz zu Medikamentenstudien häufig nicht verblindet durchgeführt wurden und dass nicht zuletzt auch der nicht verblindete Studienarzt einer Entscheidungstendenz zu Gunsten des Sponsors unterliegt. Im Folgenden sollen ungeachtet dessen einige Studien vorgestellt werden, in denen bestimmte Inhaler miteinander verglichen,
Tab. 1 Patientenangaben zu den Wunschcharakteristika bezüglich eines idealen Inhalers [22].

\begin{tabular}{|l|l|}
\hline Inhaler-Charakteristikum & \% Angabe \\
\hline leichte Bedienbarkeit während eines Asthmaanfalls & 82,8 \\
\hline Dosiszähler mit Restdosisangabe & 62,1 \\
\hline leichte Bedienbarkeit & 60,9 \\
\hline leicht zu erlernende Anwendbarkeit & 59,2 \\
\hline Hygieneaspekt & 58,0 \\
\hline komfortables Mundstück & 53,3 \\
\hline leicht zu transportieren & 40,8 \\
\hline Fühlbarkeit der inhalierten Dosisabgabe & 38,5 \\
\hline angenehmer Geschmack & 35,5 \\
\hline Möglichkeit des Recyclings & 34,3 \\
\hline Feuchtigkeitsschutz & 33,3 \\
\hline leichtes Gewicht & 25,4 \\
\hline Möglichkeit der diskreten Anwendung & 24,3 \\
\hline kleine Größe & 23,7 \\
\hline integrierte Verpackung & 22,5 \\
\hline Wiederbefüllbarkeit & 20,7 \\
\hline ansprechendes Design & 11,2 \\
\hline
\end{tabular}

Anwendungsfehler protokolliert und die Patienten nach ihrer Präferenz befragt wurden. Nicht alle Studien verwendeten dabei kontrollierte Fragebögen wie die nachfolgend dargestellten.

\section{Feeling of Satisfaction with Inhaler (FSI-10)-Fragebogen}

Der FSI-10 ist ein Fragebogen, der von den Patienten ohne weitere Unterstützung ausgefüllt wird. Er enthält 10 Fragen, die entsprechend ihrer Wichtigkeit mit 1-5 Punkten in abfallender Wertigkeit kategorisiert werden. 50 Punkte ergeben somit die bestmögliche Bewertung und 10 die schlechteste. Mit diesem Fragebogen wird der Zufriedenheitsgrad mit dem Inhaler einschließlich der Bedienbarkeit, der Leichtigkeit/Schwere ihn zu transportieren und der Anwendungsleichtigkeit/-schwere quantifiziert. Eine spanische Arbeitsgruppe belegte in einer Studie zur Vergleichsbeurteilung vom Turbohaler, dem Accuhaler und dem Novolizer, dass der FSI-10 einfach anzuwenden ist und statistisch verlässliche Ergebnisse erbringt [24]. Der Test steht in Englisch und Spanisch zur Verfügung.

\section{Patient Satisfaction and Preference Questionnaire (PASAPQ)}

Der PASAPQ umfasst 3 Fragenkomplexe mit insgesamt 13 Einzelfragen: Leistung/technische Aspekte, Verbraucherfreundlichkeit und generelle Bewertung des Inhalers, und wurde für Asthmaund COPD-Patienten von Fokusgruppen, medizinischen Experten und anhand einer Literaturrecherche entwickelt und evaluiert. [25]. Die Bewertung der einzelnen Fragen erfolgt anhand einer Likert-Skala von 1 (beste Benotung) bis 7. Die letzte Frage der generellen Bewertung bezieht sich auf die Bereitschaft, den Inhaler zukünftig weiter verwenden zu wollen ( $0=$ nein bis $100=$ absolute Zustimmung). Der PASAPQ ist in Englisch und Deutsch verfügbar. Der PASAPQ ist der einzige Test zur Quantifizierung der Patientenzufriedenheit mit Inhalern, in dem eine Angabe zur minimal klinisch-signifikanten Sensitivität gemacht wird [26,27]. Eine Punktedifferenz von mehr als 8 bis 10 Punkte in den Einzeldomainen und im Gesamtpunkte-Score wird als klinisch minimal bedeutsame Differenz angenommen [28]. 


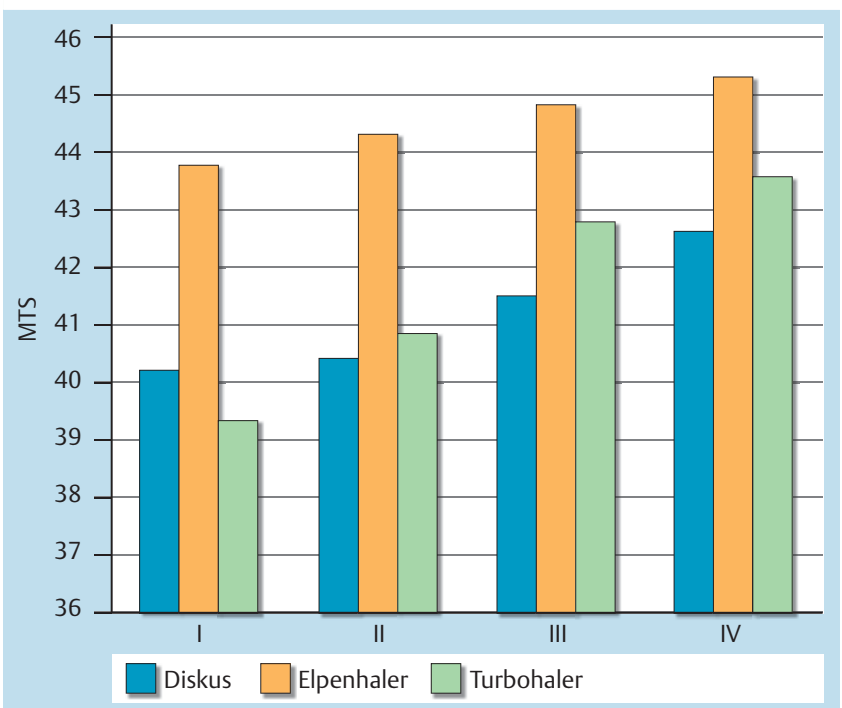

Abb. 2 Vergleich der Patientenzufriedenheit des Elpenhalers, des Diskus und des Turbohalers in Abhängigkeit vom COPD-Schweregrad unter Verwendung des 50 Punkte umfassenden FSI-10-Fragebogens [40].

\section{Onset of Effect Questionnaire (OEQ)}

Der OEQ wird vom Patienten ohne fremde Hilfe ausgefüllt. Wie der Name schon sagt, misst er den von Asthmapatienten empfundenen Wirkungsbeginn einer inhalativen Therapie $[29,30]$. Er umfasst 5 Fragen: generelle Wirkung des Medikaments, sofortiger Wirkungsbeginn, Gefühl, dass das Medikament durch eine physisch spürbare Veränderung wirkt, Wirkungsbeginn so schnell wie das Notfallmedikament, Zufriedenheit mit dem Wirkungsbeginn. Die Bewertung erfolgt anhand einer 5-stufigen Likkert-Skala von 1 (volle Zufriedenheit) bis 5 (fehlende Zufriedenheit).

\section{Patient Satisfaction Measure for Asthmatics (PASAM)}

Der PASAM umfasst 4 Fragenkomplexe: Technische Inhaleraspekte, Vergleich mit anderen Asthmamedikamenten, Gesamteindruck und Therapieeffektivität. Der Test besitzt eine hohe interne Konsistenz, vermeidet Wiederholungen und ist verlässlich in der Gruppe der kontrollierten Asthmagruppe. Die Vortestwahrscheinlichkeit war akzeptabel. Die Übereinstimmung von PASAM mit dem Asthma Quality of Life Fragebogen (AQLQ) war niedrig bis moderat. Patienten, die in die gut kontrollierte Asthmagruppe fielen, hatten die höchste PASAM-Punkteanzahl [31,32].

\section{Asthma Treatment Satisfaction Measure (ATSM)}

Der ATSM besteht aus 4 Fragekomplexen, die wiederum 11 Aspekte zur Asthmatherapie enthalten: Erwartungen an die Asthmatherapie, Behandlungspräferenz, Behandlungsergebnis aus Patientensicht und Therapiezufriedenheit. Die interne Konsistenz der Patientenerwartungen an die Asthmatherapie, das Therapieergebnis und die Patientenzufriedenheit betrugen $0,73,0,82$ und 0,95. Das ATSM-Ergebnis diskriminierte im ACO-Fragenbogen (Asthma Control Questionnaire) zwischen den kontrollierten und den nicht kontrollierten Asthmapatienten $(\mathrm{p}<0,001)$, der Selbsteinschätzung des Asthmas (mild, moderat, schwer, $\mathrm{p}<$ $0,001$ ) und dem Gesundheitsstatus ( $p<0,001)$. Im Falle einer Therapieumsetzung wurde diese durch den ATSM in 4 von 11 Attributen der jeweiligen Fragekomplexe jedoch nicht im Summationsscore als statistisch different $(\mathrm{p}<0,05)$ erfasst [33].
Treatment Satisfaction with Medicines Questionnaire (SATMED-Q)

In den SATMED-Q wurde nach einer Literatursuche und nach einem Entscheidungsprozess innerhalb eines 6 Pneumologen und 21 Patienten (von 4 Fokusgruppen) umfassenden Gremiums 17 Fragen eingeschlossen, die 6 Dimensionen abdecken: Behandlungseffektivität, Anwendungsfreundlichkeit des Medikaments, Einfluss der Medikation auf die täglichen Aktivitäten, medizinische Versorgung, Gesamtzufriedenheit und unerwünschte Nebeneffekte [34]. Dem Test wurde bescheinigt, dass er die Patientenzufriedenheit verlässlich erfassen kann. Allerdings ist unklar, mit welcher Sensitivität der Test zwischen verschiedenen Therapien stratifizieren kann.

\section{Treatment Satisfaction with Medicines Questionnaire (SAT-Q)}

Der SAT-Q enthält 26 Fragen, die sich in 4 Themen (Domainen) aufgliedern: Therapieeffektivität, Medikation, Leichtigkeit/ Schwere der Inhaleranwendbarkeit, Nebenwirkungen, Sorgen/ Bedenken [34 - 37]. Der Test hat einen akzeptablen internen Konsistenzwert und eine gute Wiederholungstestgüte. Die Testverlässlichkeit beträgt zwischen 0,66 und 0,74, die Korrelation zwischen den Skalen war moderat bis hoch. Signifikante Korrelationen fanden sich zwischen den einzelnen Domainen, dem Gesamtscore und der Patientenzufriedenheit [38].

\section{Patient Device Experience Assessment (PDEA)}

Der PDEA quantifiziert den Patienteneindruck mit einem Inhaler. Er enthält Fragen zur Patientenzufriedenheit, Bedienerfreundlichkeit des verwendeten Inhalers, der Leichtigkeit/Schwere den Inhaler anzuwenden und zu technischen Aspekten des Inhalers [39]. Die 25 Fragen werden von den Patienten, je nach Frage unterschiedlich, mit einer 5-, 6- oder 7-stufigen Likert-Skala bewertet, wobei 1 der höchsten Zustimmung entspricht. Es ist möglich, die Einzeldomaine des Fragebogens als primäre oder andere als sekundäre Studienendpunkte festzulegen.

\section{Elpenhaler}

Der Elpenhaler wurde in einer Studie unter Verwendung des 50 Punkte umfassenden FSI-10-Fragebogens auf Patientenzufriedenheit im Vergleich zum Diskus und Turbohaler bei 560 Asthma-Patienten getestet. Die durchgeführte Studie kam zu folgenden Ergebnissen: Diskus 41,4 6,1, der Elpenhaler 44,1+5,3 und der Turbohaler 43,4+5,3, wobei der Elpenhaler von den AsthmaPatienten am besten beurteilt worden ist [40]. Eine weitere, mit dem gleichen Studiendesign bei 561 COPD-Patienten durchgeführte Studie kam zu ähnlichen Ergebnissen. Danach erreichte der Diskus $40,8 \pm 6,9$, der Elpenhaler $44,7 \pm 4,4$ und der Turbohaler $41,4 \pm 5,8$ Punkte, womit der Elpenhaler unabhängig vom COPDSchweregrad am besten abschnitt ( $\bullet$ Abb.2) [40]. In einer anderen Studie wurde der Elpenhaler gegenüber dem Diskus in Bezug auf die Fehlerhäufigkeit und die Patientenpräferenz getestet. Dabei wurden 113 Patienten, die an einem Asthma oder einer COPD litten, aufgefordert, die Packungsbeilage zu lesen, wonach sie versuchen mussten, beide DPIs korrekt anzuwenden. $17 \%$ in der Diskus-Gruppe und 35\% in der Elpenhaler-Gruppe machten mindestens einen kritischen Fehler. 73\% bevorzugten den Diskus und 27\% den Elpenhaler. In der 5-stufigen Zufriedenheitsskala erhielt der Diskus die Note 1,59 und der Elpenhaler 2,48 [41]. Offenbar ist das Ergebnis von der Güte der Einweisung in das Gerät abhängig. 


\section{Diskus}

Im Vergleich zu einem pMDI bevorzugten Asthmapatienten den Diskus in 11 von 14 Domains. Positiv fielen der Dosiszähler (81,2\%), die leichte Anwendbarkeit während eines Asthmaanfalls (79,2\%), die leichte generelle Anwendbarkeit (77,1\%) und die handliche Größe (70,8\%) ins Gewicht. Für diese Studie wurden Asthmapatienten im Alter von $\geq 12$ Jahre eingeschlossenen, die mindestens mit einem kurzwirksamen $\beta 2$-Mimetikum (pMDI) und einem inhalativen Kortikosteroid (ICS als pMDI) behandelt wurden [42].

In einer anderen, an 72 Patienten (Asthma und COPD) durchgeführten Untersuchung wurden der Diskus mit dem Cyclohaler, dem Jethaler, den Benosid N-Inhaler, dem Novolizer und dem Easyhaler verglichen, wobei die Patienten einmal nach Lesen des Beipackzettels (=erste Anwendung) und einmal nach einer Einweisung ( $=2$. Anwendung) alle Inhaler testen mussten. Nach den Tests sollten sich die Patienten auf ihren bevorzugten Inhaler festlegen. Die Fehlerhäufigkeit verteilte sich wie folgt (erste/ zweite Anwendung): Diskus 38,9\%-25\%/8,3\%-13,9\%, Clickhaler $50,0 \% / 52,8 \%$, Cyclohaler 58,3\%/13,9\%, Jethaler 66,7\%/30,6\%, Benosid N Inhaler 52,8\%/22,2\%, Novolizer 52,8\%/25,0\%, Easyhaler $72,2 \% / 47,2 \%$. Die Einweisung und Erklärung des Inhalers verringerte die nur durch Lesen des Beipackzettels immer noch hohe Fehlerrate. Die Fehlerrate korrelierte negativ mit der Präferenz. Die Geräte, die am einfachsten zu bedienen waren und folglich die niedrigste Fehlerrate generierten, waren bei den Testpersonen am beliebtesten. In dieser Untersuchung waren es der Diskus und der Clickhaler (Bewertungsskala von 1 [gut] bis 7 [schlecht]): Diskus 2,21-2,02, Clickhaler 2,21, Cyclohaler 2,8, Jethaler 3,16, Novolizer 2,33, Easyhaler 2,37, Benosid N Inhaler 2,43 [43].

Serra-Batlles et al. verglichen den Diskus mit dem Turbohaler an 169 DPI-naiven Asthma- und COPD-Patienten. Ohne Einweisung ergab sich mit beiden die gleiche Fehlerhäufigkeit (ca. 30\%). Mit einer Einweisung lag diese deutlich niedriger: $7 \%$ beim Diskus und $10 \%$ beim Turbohaler. In dieser Untersuchung bevorzugten die Patienten den Diskus gegenüber dem Turbohaler. Dafür wurden vor allem folgende Gründe genannt (Diskus/Turbohaler): Schnell zu lernen (40,2\%/24,9\%), leichte Anwendung (45,6\%/ $27,2 \%)$, kleine Größe (21,3\%/42,0\%), Zählwerk mit Angabe der noch verfügbaren Dosen (87,6\%/2,4\%), Design (56,2\%/10,1\%) [22].

Im Vergleich zwischen dem Diskhaler und dem Diskus schnitt nach einer 12-wöchigen Testphase der Diskus besser ab. Ein hohes Maß der Gesamtzufriedenheit gaben $72 \%$ der Patienten beim Diskhaler und $87 \%$ beim Diskus an. Zur Frage des komfortablen Umgangs lagen die positiven Angaben bei 79\% bzw. 85\%. Am Studienende bevorzugten 61,4\% der Testpersonen den Diskus und $25,4 \%$ den Diskhaler, während sich $13,2 \%$ weder für den einen noch für den anderen Inhaler entscheiden konnten [44].

\section{Respimat}

Im Vergleich zwischen dem Respimat (Misthaler) und dem Turbohaler bevorzugten die meisten der eingeschlossenen $153 \mathrm{~Pa}-$ tienten mit einem mittelschweren bis schweren Asthma den Respimat. Das Interessante an dieser Studie ist das randomisierte, double-dummy Parallelgruppen-Studiendesign, wobei der standardisierte PASAPQ-Fragebogen angewendet wurde, um die Patientenzufriedenheit zu erfassen. So erhielt jeder Patient beide Inhaler, die beide vergleichend zu bewerten waren. Es ergab sich ein Gesamt-Punktescore von 85,5 (von 100 maximal möglichen) für den Respimat und 76,9 für den Turbohaler ( $p<0,0001$ ). $74 \%$ der Patienten bevorzugten den Respimat und nur 17\% den Turbo- haler, während sich $9 \%$ für keinen der beiden entscheiden konnten. Die Bereitschaft, ihren Inhaler weiter anzuwenden, war beim Respimat mit 80 Punkten höher als den 62 Punkten beim Turbohaler [26].

Aber auch COPD-Patienten bevorzugen den Respimat. Im Vergleich zwischen dem Respimat und dem pMDI (beide enthielten Ipratropiumbromid und Salbutamol) bewerteten die 470 COPDPatienten nach 48 Studienwochen den Respimat im PASAPQ-Fragebogen im Schnitt um ca. 10 Punkte höher. Die Zeit bis zur ersten COPD-Exazerbation war allerdings nicht statistisch signifikant different, auch wenn die Studienabbrecher und die Verweigerung, die bisherige Therapie weiter fortzuführen, in der Respimatgruppe geringer war. Bezüglich der Nebenwirkungen waren beide Gruppen äquivalent [25].

Im Vergleich vom Respimat zum Diskus fielen die Unterschiede zwar immer noch statistisch signifikant, aber zahlenmäßig im Fragebogenscore etwas geringer zu Gunsten des Respimats aus. Im PASAPQ-Punktescore wurden folgende Punkte vergeben (Respimat/Diskus/p-Wert im Gruppenvergleich): Gesamt-Punktescore $(80,2 / 75,5 / \mathrm{p}<0,001)$, Performance $(81,6 / 75,5 / \mathrm{p}<0,001)$ und Leichtigkeit der Anwendung (Convenience: 78,6/75,4/p< 0,035) [45].

Zusammenfassend wurde in drei verschiedenen Studien belegt, dass die Patienten den Respimat gegenüber dem pMDI, dem Turbohaler und dem Diskus bevorzugten [46-48].

\section{Novolizer}

Auch der Novolizer wurde in Bezug auf die Patientenmeinung positiv bewertet und wird im Zusammenhang mit einer guten Patientenadhärenz als guter Inhaler angesehen [43, 49 - 55]. Voshaar et al. fanden in einer retrospektiven Analyse an insgesamt 2444 Asthmapatienten heraus, dass nach einem Jahr noch 89\% der mit dem Novolizer und 85\% der mit dem Turbohaler behandelten Patienten bei ihrem Inhaler geblieben sind, d.h. die Novolizer-Gruppe wechselte seltener temporär oder permanent ihren Inhaler [53]: 14,7\% wechselten in der Novolizer- und 20,8\% in der Turbohaler-Gruppe $(\mathrm{p}=0,0002)$.

In einer anderen Vergleichsuntersuchung, die mit verschiedenen Inhalern an 72 Patienten (Asthma und COPD) durchgeführt wurde, schnitt der Novolizer allerdings weniger gut ab und rangierte in der Patientengunst nach dem Diskus, dem Click-, Cyclo- und dem Jethaler eher im hinteren Mittelfeld. Die Fehlerhäufigkeit mit dem jeweiligen Inhaler korrelierte mit der Inhalerpräferenz [43]. In der Summe wurde der Novolizer von ärztlicher Seite als ein gutes Inhalationsgerät eingeschätzt [49-52].

\section{Turbohaler}

Der Turbohaler ist neben dem Diskus einer der am häufigsten verschriebenen DPIs und wurde auch entsprechend häufig evaluiert. Welch et al. untersuchten in 2 jeweils einmonatigen Perioden den Turbohaler gegen 3 verschiedene pMDIs (Aerobid-M, Flovent, Vanceril Double Strength) und befragten die Patienten, die alle pMDI-Erfahrung besaßen, unter Verwendung des PDEAFragebogens nach ihrem bevorzugten Inhalationsgerät [39]. Die meisten Patienten präferierten in dieser Studie den Turbohaler gegenüber den pMDIs, da dessen Anwendung leichter und schneller erlernt werden konnte $(p<0,001)$. Die Zeit bis zur vollständigen und richtigen Anwendung betrug beim Turbohaler im Mittel 3,67 und bei den pMDIs 5,33 Minuten. Insgesamt waren die Patienten mit dem Turbohaler im PDEA-Fragebogen deutlich zufriedener $(p<0,0001)$ [39]. Ein ähnliches Ergebnis erbrachte der Vergleich zwischen dem Turbohaler (Budesonid) und einem 
mit Triamcinolon gefüllten pMDI, in dem der Turbohaler von den 945 Asthmapatienten besser bewertet wurde [56].

Ob ein Inhaler bevorzugt wird oder nicht, hängt in Studien im Wesentlichen vom Komparator ab, wie oben in den RespimatTurbohaler-Vergleichsstudien zu sehen ist, in denen der Turbohaler schlechter abschnitt [26]. In einer koreanischen Asthmastudie wendeten die Patienten den Turbohaler in nur 10,9\% fehlerfrei an, während es bei dem im Vergleich getesteten Swinghaler, der in Deutschland nicht verfügbar ist, 16,1\% waren. Allerdings war der Unterschied nicht statistisch signifikant. Die Studienteilnehmer bevorzugten allerdings den Swinghaler gegenüber dem Turbohaler (78,5\% vs. 55,0\%, p<0,001) [57].

Eine andere Studie verglich die Effektivität und Patientenakzeptanz vom Turbohaler mit dem Easyhaler, die beide Budesonid enthielten. Es wurden 326 Steroid-naive Asthmapatienten eingeschlossen. Nach einer Behandlungsphase von 8 Wochen ergaben sich keine Unterschiede im morgendlichen Peak-Flow, aber die Patienten würden, wenn sie die Wahl hätten, eher den Easyhaler als den Turbohaler als ihren bevorzugten Inhaler wählen [58]. Diese Studie wurde durch eine andere, sehr ähnlich konzipierte Untersuchung bestätigt [59]. Im Vergleich zwischen dem Turbohaler und dem Aerolizer (beide enthielten Formoterol) ergab sich bei den untersuchten nicht vollständig kontrollierten mit ICS behandelten Asthmapatienten unter dem Aerolizer eine Peak-Flow-Verbesserung um 13,861/Minute. 98\% der mit dem Aerolizer behandelten Patienten machten keine Anwendungsfehler, während es beim Turbohaler mit $86 \%$ etwas weniger waren. Ähnlich wie auch bei den anderen Inhalerstudien korrelierte die geringere Fehlerquote mit der höheren Inhalerakzeptanz der Testpersonen [60]. Wie schon erwähnt gibt es Hinweise, dass der Turbohaler in Bezug auf die Inhalerakzeptanz auch dem Diskus unterlegen ist [22]. Allerdings gibt es auch eine Untersuchung, in der Patienten zwar mit dem Turbohaler mehr Anwendungsfehler machten als mit dem Diskus, ungeachtet dessen aber angaben, ihn im Vergleich zu bevorzugen [61].

\section{Easyhaler}

Der Easyhaler ist schon nach einer Anwendungsdemonstration leicht zu benutzen, wie eine ungarische Real-Life-Studie in einer Doppeluntersuchung bei Asthma- und COPD-Patienten herausfand. Danach konnten bis 98\% aller Erwachsenen, bis 99\% aller älteren Patienten und Jugendlichen den Inhaler korrekt anwenden. $87 \%$ aller Ärzte fanden, dass die Anwendung des Easyhalers leicht gelehrt werden kann [62]. In der schon zitierten vergleichenden Untersuchung von insgesamt 7 DPIs schnitt der Easyhaler in der Patientengunst allerdings am schlechtesten ab [43]. Schweisfurth et al. und Tukiainen et al. fanden dagegen beide eine Überlegenheit gegenüber dem Turbohaler [58,59]. Damit wurde eine frühere Studie bestätigt, die bei Asthmapatienten ebenfalls eine Wirkungsäquivalenz, aber eine Patientenpräferenz zugunsten des Easyhalers (im Vergleich zum Turbohaler) fand [63]. In Bezug auf den Mundsoor und die Präferenz ist der Easyhaler den pMDIs überlegen [64]. Wettengel et al. zeigten, dass der Easyhaler bei klinischer Wirkungsäquivalenz den Asthmapatienten besser als der Diskus gefällt [65].

\section{Breezhaler}

Der Breezhaler ist ein relativ neuer Inhaler und wird ausschließlich bei COPD-Patienten eingesetzt. Auch er wurde hinsichtlich der Anwendungsfehler und Patientenakzeptanz getestet. $82 \mathrm{~Pa}$ tienten mit einer milden bis schweren COPD erhielten in einer 7-Tage cross-over Studie entweder den Breez- oder den Handihaler. Die Patienten wurden mittels einer Beschreibung und einer kurzen Trainingsphase in die Inhaler eingewiesen. In beiden Gruppen wendeten $>77 \%$ aller Patienten ihren Inhaler korrekt an. Während $61 \%$ den Breezhaler bevorzugten, sprachen sich nur $31 \%$ für den Handihaler aus $(p=0,01)$ [66].

\section{Handihaler}

Der Handihaler ist ein schon lange auf dem Markt befindlicher, für die COPD-Therapie verwendeter DPI. Im Vergleich mit einem pMDI wies er eine geringere Fehlerquote auf, wie Dahl et al. in einer 139 COPD-Patienten umfassenden Studie nachwiesen [67]. Vier Wochen, nachdem die Inhaler den Patienten erklärt wurden, wiesen die Patienten mit dem Handihaler weniger Fehler auf $(35,3 \%)$ als diejenigen, die mit dem pMDI behandelt wurden $(15,1 \%)$. Die Autoren folgerten, dass die Anwendung des Handihalers leicht gelehrt werden kann und dass damit weniger Anwendungsfehler auftreten würden als mit einem pMDI [67]. Allerdings scheint der Handihaler in der Patientengunst dem Diskus und neueren DPIs, wie z.B. dem Breezhaler oder dem Genuair, unterlegen zu sein [66,68,69].

\section{Genuair}

Der Genuair wurde mit dem Handihaler bei insgesamt 105 Patienten, die an einer mittelschweren bis schweren COPD litten, in einer randomisierten, open-label, multizentrischen crossover Studie verglichen. Nach einer Therapiephase von 2 Wochen bevorzugten $79,1 \%$ aller Patienten den Genuair gegenüber dem Handihaler $(20,9 \%, \mathrm{p}<0,0001)$. In der 5-stufigen Zufriedenheitsskala rangierte der Genuair mit 4,6 Punkten ebenfalls vor dem Handihaler, der 3,8 Punkte erhielt $(p<0,0001)$. Die Patienten waren auch eher willig, den Genuair weiter zu benutzen als den Handihaler (84,0 gegenüber 62,5 Punkten, $p<0,0001)$. Parallel dazu machten nur 2,9\% aller Testpersonen mehr als einen kritischen Fehler, der zu einem Wirkungsverlust des Inhalers geführt hätte, als mit dem Handihaler (19,0\%, p<0,0001) [69].

\section{Ellipta}

Der Ellipta Inhaler ist ein neuer DPI, der bei Patienten mit einem Asthma oder einer COPD untersucht wurde. Svedsater et al. führen an amerikanischen Asthma- $(\mathrm{n}=33)$ und COPD-Patienten $(\mathrm{n}=$ 42) ein strukturiertes 45-minütiges Telefoninterview durch, in dem diese nach ihren Erfahrungen im Vergleich zu den bisher von ihnen verwendeten Inhalern befragt wurden. 71\% der Asthmapatienten bevorzugten Ellipta gegenüber dem Diskus und $60 \%$ gegenüber einem pMDI. Unter den COPD-Patienten präferierten 86\% Ellipta gegenüber dem Diskus, 95\% gegenüber dem Handihaler und $85 \%$ gegenüber einem pMDI. Die Gesamtbewertung der Anwendung des neuen DPI fiel mit >9 Punkten (von 10) bei beiden Patientengruppen positiv aus [70]. 
Tab.2 Inhaler, die auf dem deutschen Markt verfügbar sind (Stand 05/2014). LABA= Langwirksame $\beta 2$-Sympathomimetika, LAMA = Langwirksame Anticholinergika, ICS = Inhalative Kortikosteroide, SABA = Kurzwirksame $\beta 2$-Sympathomimetika.

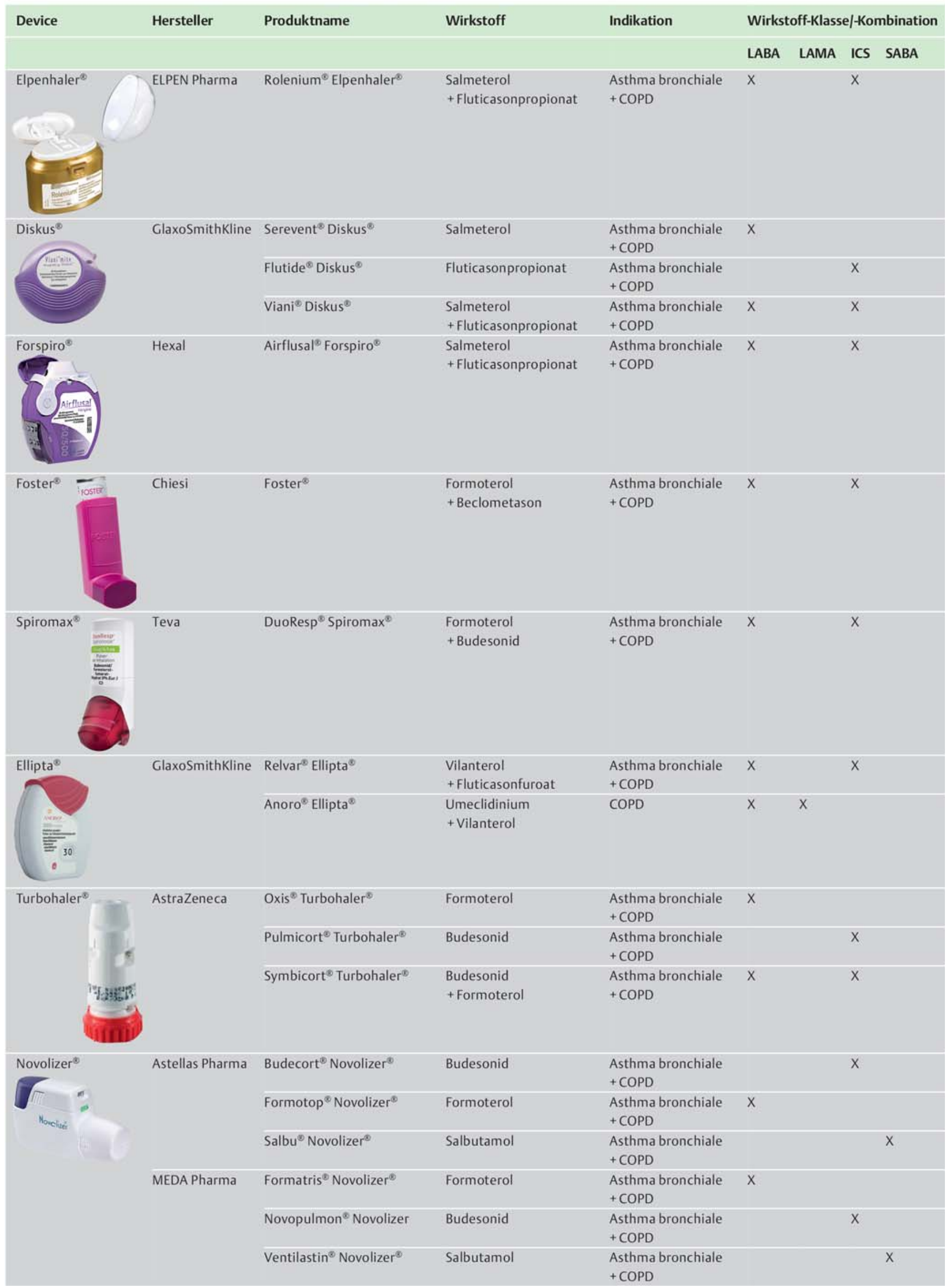


Tab.2 (Fortsetzung)

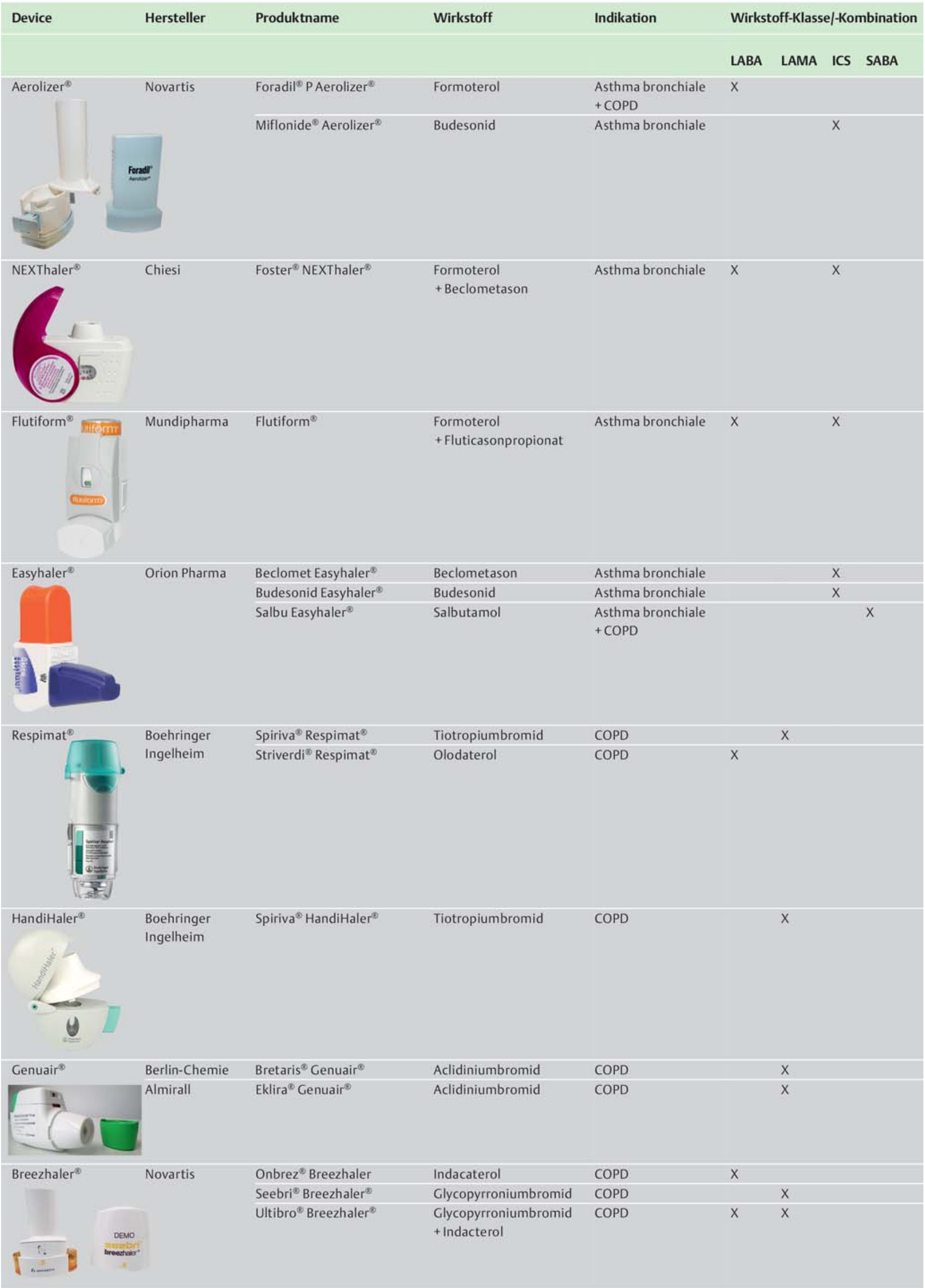


Fazit

Nur die korrekte Anwendung des Inhalers garantiert auch die Wirksamkeit des zu inhalierenden Medikaments in den Bronchien. Die inhalative Therapie ist generell schwieriger als eine orale Medikamentengabe. Daher werden an Inhaler eine Fülle von Forderungen gestellt, die die korrekte und angenehme Einnahme sowie die Vermeidung von Anwendungsfehlern sicherstellen sollen. In Leitlinien und auch bei der Verschreibung von Inhalern werden häufig die Bedürfnisse und Neigungen der Patienten zu wenig beachtet. Vergleichsuntersuchungen mit mehreren Inhalern haben jedoch gezeigt, dass Patienten sehr wohl eine spezifische Meinung darüber gewinnen können und haben, welcher Inhalertyp für sie am besten geeignet ist. Somit endet die Asthma- und COPD-Therapie nicht in der Einschätzung der Wirksamkeit verschiedener Medikamente, sondern muss neben dem Inhalertraining auch die Bedürfnisse und Fähigkeiten der Betroffenen miteinschließen. Denn erst, wenn der Patient seinen Inhaler in den Grundzügen technisch versteht, ihn korrekt anwenden kann und ihn auch akzeptiert, ist von einer guten Patientenadhärenz auszugehen.

\section{Interessenkonflikt}

\section{$\nabla$}

A. Gillissen: Erstattung von Reisekosten oder Teilnahmegebühren für einen Kongress oder eine Fortbildungsveranstaltung von Almirall, Elpen, Berlin-Chemie, Boehringer-Ingelheim, Chiesi, GlaxoSmithKline, Novartis; Honorar für einen Vortrag von Almirall, Berlin-Chemie, Boehringer-Ingelheim, GlaxoSmithKline, Novartis, Chiesi; Unterstützung für ein Forschungsvorhaben oder finanzielle Zuwendung für die Teilnahme an einer Studie von Novartis; Honorar für Beratertätigkeit von Almirall, BerlinChemie, Boehringer-Ingelheim, Chiesi, Elpen, Novartis.

\section{Literatur}

1 Voshaar Th, App EM, Berdel D et al. Empfehlungen für die Auswahl von Inhalationssystemen zur Medikamentenverabreichung. Pneumologie 2002; 55: 579-586

2 Gillissen A. Patient adherence in asthma. J Physiol Pharmacol 2007; 58: 205-222

3 Prabhakaran L, Lim G, Abisheganaden J et al. Impact of an asthma education programme on patients' knowledge, inhaler technique and compliance to treatment. Singapore Med J 2006; 47: 225-231

4 GINA Executive and Science Committee, Hrsg. Global Initiative for Asthma (GINA). Global strategy for asthma management and prevention;2014

5 GOLD Executive Committee. Global initiative for chronic obstructive lung disease. 2014: www.goldcopd.com

6 British Thoracic Society. The british guidelines on asthma management. Asthma in adults and schoolchildren. Thorax 2003; 58: 1-94

7 Dolovich MB, Ahrens RC, Hess DR et al. Device selection and outcomes of aerosol therap. Evidence-based guidelines: American Chest Physicians/American College of Asthma. Chest 2005; 127: 335-371

8 Palen van der J, Klein JJ, Herwaarden van C LA et al. Multiple inhalers confuse asthma patients. Eur Respir J 1999; 14: 1037

9 Gillissen A, Juergens UR, Büsch K. Therapieaadhärenz bei Asthma bronchiale. Methoden zur Verbesserung. Dtsch Med Wschr 2008; 133 : $1451-1456$

10 Molimard M. How to achieve good compliance and adherence with inhalation therapy. Curr Med Res Opin 2007; 21: 33 -S37

11 Virchow JC, Crompton GK, Dal NR et al. Importance of inhaler devices in the management of airway disease. Respir Med 2008; 102: 10-19
12 Capstick TG, Clifton IJ. Inhaler technique and training in people with chronic obstructive pulmonary disease and asthma. Expert Rev Respir Med 2012; 6: $91-101$

13 Molimard M, Raherison C, Lignot S et al. Assessment of handling of inhaler devices in real life: an observational study in 3811 patients in primary care. J Aerosol Med 2003; 16: 249-254

14 Knipel V, Criée CP, Windisch $W$. Korrekte Inhalationstherapie: Einweisung mittels Internet-verfügbarer Filmsequenzen. Pneumologie 2013; 67: $157-161$

15 Wieshammer S, Dreyhaupt J. Dry powder inhalers: which factors determine the frequency of handling errors? Respiration 2008; 75: 18-25

16 Small M, Anderson P, Vickers A et al. Importance of inhaler-device satisfaction in asthma treatment: real-world observations of physicianobserved compliance and clinical/patient-reported outcomes. Adv Ther 2011; 28: 202-212

17 Rau JL. Practical problems with aerosol therapy in COPD. Respir Care 2006; 51: $158-172$

18 Bender B, Milgrom $H$, Apter A. Adherence intervention research: what have we learned and what do we do next? J Allergy Clin Immunol 2003; 112: 489-494

19 Murphy KR, Bender BG. Treatment of moderate to severe asthma: patient perspectives on combination inhaler therapy and implications for adherence. J Asthma Allergy 2009; 2: 63 - 72

20 Giraud V, Roche N. Misuse of corticosteroid metered-dose inhaler is associated with decreased asthma stability. Eur Respir J 2002; 19: $246-251$

21 Rand CS, Wise RA, Nides $M$ et al. Metered-dose inhaler adherence in a clinical trial. Am Rev Respir Dis 1992; 146: 1559-1564

22 Serra-Batlles J, Plaza V, Badiola $C$ et al. Patient perception and acceptability of multidose dry powder inhalers: a randomized crossover comparison of Diskus/Accuhaler with Turbuhaler. J Aerosol Med 2002; 15: $59-64$

23 Chapman D, Voshaar T, Virchow JC. Inhaler choice in primary care. Eur Respir Rev 2005; 14: 117-122

24 Perpina TM, Viejo JL, Sanchis J et al. Satisfaccion y preferencia del paciente asmatico por los dispositivos de inhalacion. Aplicacion del FSI-10. Arch Bronconeumol 2008; 44: 346-352

25 Ferguson GT, Ghafouri M, Dai $L$ et al. COPD patient satisfaction with ipratropium bromide/albuterol delivered via Respimat: a randomized, controlled study. Int J Chron Obstruct Pulmon Dis 2013; 8: 139-150

26 Hodder R, Reese PR, Slaton T. Asthma patients prefer Respimat Soft Mist Inhaler to Turbuhaler. Int J Chron Obstruct Pulmon Dis 2009; 4: 225232

27 Kozma CM, Slaton TL, Monz BU et al. Development and validation of a patient satisfaction and preference questionnaire for inhalation devices. Treat Respir Med 2005; 4: 41 - 52

28 Kilfeather SA, Ponitz HH, Beck E et al. Improved delivery of ipratropium bromide/fenoterol from Respimat Soft Mist Inhaler in patients with COPD. Respir Med 2004; 98: 387-397

29 Hauber AB, Mohamed AF, Johnson FR et al. Quantifying asthma patient preferences for onset of effect of combination inhaled corticosteroids and long-acting beta2-agonist maintenance medications. Allergy Asthma Proc 2009; 30: 139-147

30 Mohamed AF, Hauber AB, Johnson FR et al. Patient preferences and linear scoring rules for patient-reported outcomes. Patient 2010; 3 : 217-227

31 Mathias JM. Rounding tool off to a good start in improving patient satisfaction. OR Manager 2014; 30: 1, 6, 8-1, 6, 9

32 Mathias SD, Warren EH, Colwell HH et al. A new treatment satisfaction measure for asthmatics: a validation study. Qual Life Res 2000; 9: $873-882$

33 Martin ML, Patrick DL, Bushnell DM et al. Development of the asthma treatment satisfaction measure. Curr Med Res Opin 2009; 25: 2495 2506

34 Ruiz MA, Pardo A, Rejas J et al. Development and validation of the "Treatment Satisfaction with Medicines Questionnaire" (SATMED-Q). Value Health 2008; 11: 913-926

35 Delestras S, Roustit M, Bedouch P et al. Comparison between two generic questionnaires to assess satisfaction with medication in chronic diseases. PLoS One 2013; 8: e56247

36 Rejas J, Ruiz M, Pardo A et al. Detecting changes in patient treatment satisfaction with medicines: the SATMED-Q. Value Health 2013; 16: $88-96$ 
37 Rejas J, Ruiz MA, Pardo A et al. Minimally important difference of the Treatment Satisfaction with Medicines Questionnaire (SATMED-Q). BMC Med Res Methodol 2011; 11: 142

38 Campbell JL, Kiebert GM, Partridge MR. Development of the satisfaction with inhaled asthma treatment questionnaire. Eur Respir J 2003; 22: $127-134$

39 Welch MJ, Nelson HS, Shapiro G et al. Comparison of patient preference and ease of teaching inhaler technique for Pulmicort Turbuhaler versus pressurized metered-dose inhalers. J Aerosol Med 2004; 17: 129-139

40 Zervas E, Gaga M, Grekas $N$ et al. Assessment of satisfaction with inhalation devices in COPD patients. Eur Respir J 2012: p758

41 van der Palen JV, Goosens M, Groothuis-Oudshoorn K et al. A randomised cross-over trial investigating the ease of use and preference of two dry powder inhalers in patients with asthma or chronic obstructive pulmonary disease. Expert Opin Drug Deliv 2013; 10: 1171 - 1178

42 Liam CK, Lim KH, Wong CM. Acceptance of the Accuhaler, a multi-dose powder inhaler, among asthmatic patients: a comparison with the pressurized metered-dose inhaler. Asian Pac J Allergy Immunol 2000; 18: $135-140$

43 Schulte $M$, Osseiran $K$, Betz R et al. Handling of and preferences for available dry powder inhaler systems by patients with asthma and COPD. J Aerosol Med Pulm Drug Deliv 2008; 21: 321-328

44 Mahajan P, Okamoto L. Patient satisfaction with the Diskhaler and the Diskus inhaler, a new multidose power delivery system for the treatment of asthma. Clin Ther 1997; 19: 1126-1134

45 Freytag $F$, Golisch $W$, Wolf $K$. COPD and asthma patients are satisfied with handling an $d$ inhalation characteristics of Soft Mist (Berodual Respimat). Eur Respir J Suppl 2006; 28: 739s

46 Hodder R, Price D. Patient preferences for inhaler devices in chronic obstructive pulmonary disease: experience with Respimat Soft Mist inhaler. Int J Chron Obstruct Pulmon Dis 2009; 4: 381-390

47 Schurmann W, Schmidtmann S, Moroni P et al. Respimat Soft Mist inhaler versus hydrofluoroalkane metered dose inhaler: patient preference and satisfaction. Treat Respir Med 2005; 4: 53-61

48 Chrystyn H. The Diskus: a review of its position among dry powder inhaler devices. J Clin Pract 2007; 61: 1022-1036

49 Crompton GK. How to achieve good compliance with inhaled asthma therapy. Respir Med 2004; 98 (Suppl. B): S35 - S40

50 Magnussen $H$. Novolizer: how does it fit into inhalation therapy? Curr Med Res Opin 2005; 21 (Suppl. 04): S39-S46

51 Virchow JC. What plays a role in the choice of inhaler device for asthma therapy? Curr Med Res Opin 2005; 21 (Suppl. 04): S19-S25

52 Virchow JC. Guidelines versus clinical practice-which therapy and which device? Respir Med 2004; 98 (Suppl. B): S28 -S34

53 Voshaar T, Kostev K, Rex J et al. A retrospective database analysis on persistence with inhaled corticosteroid therapy: comparison of two dry powder inhalers during asthma treatment in Germany. Int J Clin Pharmacol Ther 2012; 50: 257-264

54 Moeller M, Grimmbacher S, Munzel U. Improvement of asthma therapy by a novel formoterol multidose dry powder inhaler. Arzneimittelforschung 2008; 58: $168-173$
55 Below A, Bickmann D, Breitkreutz J. Assessing the performance of two dry powder inhalers in preschool children using an idealized pediatric upper airway model. Int J Pharm 2013; 444: 169-174

56 Weiss KB, Paramore LC, Liljas B et al. Patient satisfaction with budesonide Turbuhaler versus triamcinolone acetonide administered via pressurized metered-dose inhaler in a managed care setting. J Asthma 2005; 42: 769-776

57 Chang YS, Park MJ, Bai C et al. Comparative study of patients in correct usage of and preference for the Swinghaler and Turbuhaler multidose inhalers. J Asthma 2012; 49: 750-756

58 Schweisfurth H, Malinen A, Koskela T et al. Comparison of two budesonide powder inhalers, Easyhaler and Turbuhaler, in steroid-naive asthmatic patients. Respir Med 2002; 96: 599-606

59 Tukiainen H, Rytila P, Hamalainen KM et al. Safety, tolerability and acceptability of two dry powder inhalers in the administration of budesonide in steroid-treated asthmatic patients. Respir Med 2002; 96: $221-229$

60 Eliraz A, Ramirez-Rivera A, Ferranti P et al. Similar efficacy following four weeks treatment of asthmatics with formoterol 12 micrograms b. d. delivered by two different dry powder inhalers: differences in inhaler handling. Int J Clin Pract 2001; 55: 164-170

61 van der Palen J, Klein JJ, Schildkamp AM. Comparison of a new multidose powder inhaler (Diskus/Accuhaler) and the Turbuhaler regarding preference and ease of use. J Asthma 1998; 35: 147-152

62 Galffy G, Mezei G, Nemeth $G$ et al. Inhaler competence and patient satisfaction with Easyhaler(R): results of two real-life multicentre studies in asthma and COPD. Drugs R.D 2013; 13: 215-222

63 Jager L, Laurikainen $K$, Leinonen $M$ et al. Beclomethasone dipropionate Easyhaler is as effective as budesonide Turbohaler in the control of asthma and is preferred by patients. German Study Group. Int J Clin Pract 2000; 54: 368 - 372

64 Newman SP, Pitcairn GR, Adkin DA et al. Comparison of beclomethasone dipropionate delivery by easyhaler dry powder inhaler and pMDI plus large volume spacer. J Aerosol Med 2001; 14: 217-225

65 Wettengel $R$, Laurikainen $K$, Silvasti $M$ et al. Therapeutic equivalence and acceptability of two multidose powder inhalers in the treatment of asthma. Respiration 2000; 67: 77-82

66 Chapman KR, Fogarty CM, Peckitt $C$ et al. Delivery characteristics and patients' handling of two single-dose dry-powder inhalers used in COPD. Int J Chron Obstruct Pulmon Dis 2011; 6: 353-363

67 Dahl R, Backer V, Ollgaard B et al. Assessment of patient performance of the HandiHaler compared with the metered dose inhaler four weeks after instruction. Respir Med 2003; 97: 1126-1133

68 Moore AC, Stone S. Meeting the needs of patients with COPD: patients' preference for the Diskus inhaler compared with the Handihaler. Int J Clin Pract 2004; 58: 444-450

69 van der Palen J, Ginko T, Kroker A et al. Preference, satisfaction and errors with two dry powder inhalers in patients with COPD. Expert Opin Drug Deliv 2013; 10: 1023-1031

70 Svedsater H, Dale P, Garrill K et al. Qualitative assessment of attributes and ease of use of the ELLIPTA dry powder inhaler for delivery of maintenance therapy for asthma and COPD. BMC Pulm Med 2013; 13: 72 